\title{
Entrenamiento psicológico para la mejora de la atención y la autoconfianza en un futbolista
}

\author{
Aurelio Olmedilla y Julián J. Dominguez-Igual \\ Universidad de Murcia, España
}

RESUMEN: En este trabajo se presenta una intervención psicológica con un jugador de fútbol profesional que muestra un nivel muy bajo de confianza y problemas para poder concentrarse durante las competiciones. Desde un enfoque cognitivo-conductual se implementó un programa de entrenamiento psicológico con una duración de cuatro meses. Se realizó una evaluación inicial y final para valorar la satisfacción y la eficacia del programa. La intervención estuvo formada por técnicas y estrategias como reestructuración cognitiva, establecimiento de objetivos, plan de competición, auto-registros conductuales y técnicas de relajación y visualización. Al finalizar el programa el futbolista muestra un nivel mucho más alto de confianza y tiene recursos eficaces para poder concentrarse tanto en los partidos como en los entrenamientos. Se postula la adecuación del enfoque cognitivo-conductual a la intervención psicológica en el ámbito deportivo, enfatizando la importancia de incorporar estrategias psicológicas en el trabajo del entrenamiento deportivo.

PALABRAS CLAVE: Preparación mental, enfoque cognitivo-conductual, fútbol, rendimiento, CPRD.

\section{Psychological Training to Improve Attention and Self-Confidence in a Soccer Player}

ABSTRACT: This paper presents a psychological intervention with a professional soccer player with very low self-confidence and trouble concentrating during competition. With a cognitive-behavioral approach, a psychological training program lasting four months was implemented. Baseline and final evaluations were conducted to assess the satisfaction with and the effectiveness of the program. The intervention consisted of techniques and strategies such as cognitive restructuring, setting goals, competition plan, behavioral self-assessment and relaxation and visualization techniques. At the end of the program the player shows a much higher level of confidence and possesses effective resources to be able to concentrate during both games and training sessions. The adaptation of cognitive-behavioral psychological intervention to sports is considered, emphasizing the importance of incorporating psychological strategies in the work of sports training.

KEYWORDS: Mental Preparation, cognitive-behavioral approach, soccer, performance, CPRD.

\section{Treinamento psicológico para melhorar a atenção e auto-confiança em um jogador do futebol}

RESUMO: Este artigo apresenta uma intervenção psicológica com um jogador de futebol profissional, que mostra um nível muito baixo de confiança e problemas para se concentrar durante as competições. A partir duma abordagem cognitivo-comportamental, implementou-se um programa de treinamento psicológico com uma duração de quatro meses. Levou-se a cabo avaliação inicial e final para valorar a satisfação e a eficácia do programa. A intervenção consistiu de técnicas e estratégias como a reestruturação cognitiva, o estabelecimento de objetivos, plano de competição, auto-registos comportamentais e técnicas de visualização e de relaxação. No final do programa, o jogador mostra um nível de confiança muito mais elevado e tem recursos eficaces para se concentrar tanto nos jogos como nos treinamentos. Defendese a adequação da intervenção psicológica cognitivo-comportamental no esporte, enfatizando a importância de incorporar estratégias psicológicas no trabalho de treinamento esportivo.

PALAVRAS-CHAVE: preparação mental, abordagem cognitivo-comportamental, futebol, desempenho, CPRD.

Artículo recibido: 02/06/2016 | Artículo aceptado: 03/09/2016

Aurelio Olmedilla es Psicólogo, Profesor Titular del Departamento de Personalidad, Evaluación y Tratamiento Psicológico de la Universidad de Murcia. Director del Servicio de Actividades Deportivas de la Universidad de Murcia. Editor Jefe de la Revista de Psicología del Deporte.

Julián José Domínguez-Igual es Graduado en Educación Social por la Universidad de Murcia.

La Correspondencia sobre este articulo debe enviarse al primer autor al Departamento de Personalidad, Evaluación y Tratamiento Psicológico. Facultad de Psicología. Universidad de Murcia. Campus de Espinardo. 30100 Espinardo. Murcia. E-mail:

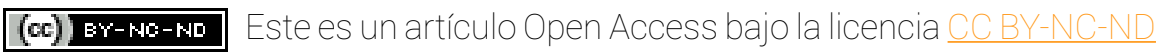


La disposición psicológica del deportista es una de las variables a tener en cuenta para el rendimiento deportivo (Buceta, 1998; Cox, 2008; Weinberg y Gould, 2010). Así, el entrenamiento psicológico, como una parte más del entrenamiento deportivo, se ha ido incluyendo en el método de trabajo de muchos deportistas y equipos deportivos (Abenza et al., 2014; Chicau, Silva y Palmi, 2012; García-Naveira y Jerez, 2012; Jaenes, Caracuel y Peñaloza, 2012; Olmedilla, Ortega, González y Serpa, 2015; Wood y Wilson, 2012). Generalmente, mediante este trabajo se persigue que el deportista aprenda habilidades y estrategias psicológicas que le permitan, por un lado, afrontar las diferentes situaciones deportivas y responder adecuadamente ante estas demandas (Buceta, López de la Llave, Pérez-Llantada, Vallejo y del Pino, 2002; Cantón, Checa y Ortín, 2009; Olivares, López y Garcés de los Fayos, 2016; Reyes, Raimundi y Gómez, 2012; Wood y Wilson, 2012), y por otro, realizar una práctica deportiva satisfactoria con una percepción de bienestar psicológico que le permita crecer y madurar como persona (Olmedilla, Ortega, Ortín y Andreu, 2008; Olmedilla, Bazaco, Ortega y Boladeras, 2011; Romero et al., 2010; Salgado, Rivas y García-Mas, 2011; Sheard y Golby, 2006).

El entrenamiento psicológico también está determinado por las características de la especialidad deportiva y del formato de trabajo psicológico a realizar (Buceta et al., 2002; Lorenzo, Gómez, Pujals y Lorenzo, 2012; Morilla et al., 2001). Olmedilla, Ortega, Boladeras, Ortín y Bazaco (2013) señalan que no es lo mismo preparar psicológicamente a un deportista que practica deporte individual, que preparar a un equipo deportivo, o preparar individualmente a un deportista que forma parte de un equipo, como es el caso que se presenta en este estudio. El psicólogo cuando trabaja con deportistas de forma individual puede tener un feedback más directo y fiable, que le permite observar la mejoría, en su caso, de diferentes aspectos psicológicos trabajados, además de la satisfacción del deportista con el trabajo y la aplicabilidad de lo aprendido en diferentes contextos; sin embargo, cuando el psicólogo trabaja con grupos o equipos deportivos puede no tener una visión tan clara del proceso de entrenamiento psicológico, apareciendo otras variables (relaciones entre los miembros, cooperación y cohesión, rendimiento individual y de equipo, resultados, etc.) que dificultan dicha percepción.

Desde la perspectiva de la práctica profesional es muy importante que el psicólogo conozca los progresos de los deportistas en el aprendizaje de las técnicas y estrategias psicológicas, y su aplicación en el contexto deportivo, o incluso extradeportivo. No sólo debe interesar al psicólogo del deporte ver si el deportista, o el equipo, rinde mejor en el cam- po o la pista, sino fundamentalmente como ha mejorado sus recursos psicológicos, su habilidad mental, en la línea de lo señalado por algunos autores respecto al control de estrés o reducción de la ansiedad (Godoy-Izquierdo, Vélez y Godoy, 2007; Junichi y Hajime, 2007; Landi, Benedetti y Merla, 2007; Munroe-Chandler, Murphy, Hall y Fishburne, 2007). En definitiva, la valoración del trabajo psicológico debe centrarse, básicamente, en la mejora del funcionamiento psicológico de los deportistas, valorando su progreso adaptativo, su relación con el rendimiento y su bienestar psicológico.

En el presente trabajo, en un proceso de pre y post evaluación e intervención, se expone el entrenamiento psicológico realizado con un futbolista de 23 años, centrado en el fortalecimiento de la autoconfianza y de la concentración. Los estudios de caso único son un método muy bueno para evaluar la eficacia de las intervenciones (Barker, Mellalieu, McCarthy, Jones y Moran, 2013; Turner y Barker, 2013), y tal y como indican Barker et al. (2013), últimamente han empezado a ser muy valorados, ya que pueden proporcionar un marco de referencia para investigadores y profesionales, sobre todo en el ámbito de la Psicología del Deporte Aplicada. Además de proporcionar un marco idóneo para mostrar la aplicación de los conceptos teóricos, y por lo tanto reducir la brecha entre teoría y práctica (Kuntz y Hesslar, 1998), suponen una muestra excelente de intervenciones en distintos aspectos del rendimiento o la salud (Abenza, Bravo y Olmedilla, 2006; Lameiras, Almeida, Pons y García-Mas, 2014; Latinjak, Font-Lladó, Zourbanos y Hatzigeorgiadis, 2016; Nieto y Olmedilla, 2001).

Partiendo de la revisión realizada, los objetivos de presente trabajo son la mejora de la capacidad de concentración y autoconfianza del deportista. La hipótesis que se plantea es: "el futbolista mejorará su capacidad de concentración y su nivel de autoconfianza tras la aplicación de un programa de entrenamiento psicológico específico".

\section{Método}

\section{Participante}

El programa de intervención se llevó a cabo con un futbolista de 23 años, que desde los 6 años practica fútbol. Ha participado con su selección autonómica en el Campeonato de España. Desde juvenil formó parte de un club de la Liga de Fútbol Profesional, llegando a jugar la fase de ascenso a $2^{\mathrm{a}} \mathrm{B}$ con el equipo filial, y participó en los entrenamientos del primer equipo. Posteriormente ha jugado en la categoría $2^{\mathrm{a}} \mathrm{B}$ y disputado la fase de ascenso a $2^{a}$ División $A$, siendo nombrado como 
el quinto mejor sub-23 de la categoría. Actualmente, juega en un club de $2^{\mathrm{a}} \mathrm{B}$, compaginando la práctica del fútbol (con 5 días de entrenamiento semanal - 2 horas cada entrenamiento -, y una competición - 2 horas - cada fin de semana) con sus estudios del Grado de Educación Social en la Universidad de Murcia, del que solo le faltan dos asignaturas para graduarse.

\section{Instrumentos y material}

Para la evaluación psicológica inicial se utilizó el Cuestionario de Características Psicológicas para el Rendimiento Deportivo (CPRD) de Gimeno, Buceta y Pérez-Llantada (2001). El CPRD es un instrumento válido y fiable que consta de 55 ítems distribuidos en cinco factores: Control de Estrés, Influencia de la Evaluación del Rendimiento, Motivación, Habilidad Mental y Cohesión de Equipo. En el presente trabajo solo se presentan los resultados de Autoconfianza y de Atención, pertenecientes a los factores Control de Estrés e Influencia de la Evaluación del Rendimiento.

Además se presentan los resultados de los aspectos conformados por los ítems específicos de cada variable analizados por el propio programa informático del CPRD, así como los derivados de la tolerancia al bajo rendimiento muy relacionados con los anteriores (Gimeno y Buceta, 2010). Así, se presentan los valores de la Atención en tres aspectos asociados con esta función: la habilidad para estar concentrado, la falta de habilidad general y los motivos específicos para la pérdida de concentración; los valores de la Autoconfianza en otros tres aspectos relacionados con esta variable: la confianza general, la competición y los entrenamientos; y los valores de la Tolerancia al bajo rendimiento del deportista también en tres aspectos: las malas actuaciones, los errores y las críticas.

Se ha considerado este cuestionario por ser uno de los instrumentos que mayor utilidad está mostrando en la evaluación de las características psicológicas implicadas en el rendimiento deportivo, en la continuidad en el deporte de competición y en el éxito deportivo (Gimeno y Buceta, 2010; Gimeno, Buceta y Pérez-Llantada, 2007). El CPRD se cumplimentó en dos sesiones dado que algunos de los ítems, además de ser contestados en forma de escala tipo Likert ( $0=$ totalmente en desacuerdo; 4 = totalmente de acuerdo), eran abordados con mayor profundidad para obtener una mejor información de lo que el deportista realmente pensaba, sentía o actuaba en relación a la cuestión planteada en el ítem. Esta información ayudó a la creación de registros de auto-observación y seguimiento del programa de entrenamiento psicológico.

Además del CPRD, también se utilizó un registro ad hoc (ver un ejemplo en Anexo I) para analizar las fortalezas y debilidades psicológicas percibidas por el deportista, denominado Aspectos Psicológicos Fuertes y Débiles que el depor- tista debía de cumplimentar en casa, el cual se analizó con el psicólogo posteriormente.

Para la evaluación psicológica final (evaluación post-intervención) se volvió a utilizar el CPRD y un cuestionario ad hoc de evaluación de la preparación psicológica (en la Tabla 2 del apartado de Resultados se puede ver), que servía para obtener una reflexión sobre la preparación psicológica realizada, evaluando cuantitativa y cualitativamente diferentes aspectos de la misma, lo que puede ayudar a valorar mejor la eficacia de técnicas, estrategias y procedimientos.

\section{Procedimiento y diseño de la intervención}

La demanda psicológica inicial del futbolista fue mejorar su rendimiento deportivo, concretamente mejorar su rendimiento en los partidos, ya que "no me siento un futbolista importante... me siento como agarrotado durante los partidos... no hago que ocurran cosas, me da miedo fallar". A partir de la entrevista inicial y de la cumplimentación de aspectos psicológicos fuertes y débiles ("me ocurre en los partidos que suelo distraerme con facilidad y perder la concentración, me pongo a pensar en cosas o mirar cosas realmente absurdas", "no tengo confianza en mis posibilidades, trato de no fallar en vez de arriesgarme, jugando muy cohibido"), se propuso un programa de entrenamiento psicológico centrado en la mejoría de la concentración en competición y de la autoconfianza, aspectos que preocupaban mucho al futbolista. El programa duró 4 meses, desde febrero de 2016 hasta mayo de 2016, y estuvo compuesto por 9 sesiones de 1 hora (aproximadamente) de duración cada una. El marco teórico para la implementación del programa de intervención es el propuesto por la Terapia Cognitivo Conductual, utilizada por una buena parte de los profesionales de la Psicología del Deporte (Lindern, 2016; Mostaan, Sanatkaran y Bahari, 2015) y considerando los cuatro principios clave de la misma señalados por McArdle y Moore (2012): que los sesgos sistemáticos del procesamiento de información juegan un papel crucial en el desarrollo y mantenimiento de los problemas psicológicos, que el cambio cognitivo es fundamental para un resultado terapéutico positivo, el reconocimiento de tres tipos distintos de conocimientos en diferentes niveles de procesamiento cognitivo (creencias, reglas y pensamientos automáticos), y que los diferentes problemas o trastornos se pueden diferenciar en las bases de contenido cognitivo específico.

Cabe destacar que la intervención estuvo formada por la aplicación de técnicas y estrategias como reestructuración cognitiva, establecimiento de objetivos, plan de competición, auto-registros conductuales y técnicas de relajación y visualización (Lindern, 2016; Mostaan et al., 2015). 
La secuencia del trabajo realizado fue la siguiente:

a) Evaluación psicológica inicial.

- Determinación del perfil psicológico.

b) Intervención psicológica y seguimiento.

- Explicación, aprendizaje y aplicación de técnicas y estrategias

c) Evaluación psicológica final.

- Valoración de la eficacia del programa y recomendaciones de futuro.

En la Tabla 1 se puede observar el desarrollo del programa de intervención, así como las actividades que el deportista debía de realizar en el periodo inter-sesiones.

\section{Resultados}

Se muestran agrupados en figuras los valores (percentiles según los baremos del propio cuestionario) de los factores del CPRD pertinentes a las variables Autoconfianza y Atención, correspondientes a la evaluación inicial y la final, para poder observar la diferencia entre ambos momentos, utilizando el sistema de corrección del programa informático del CPRD (Gimeno y Buceta, 2010).

En la Figura 1 se pueden observar los valores de Autoconfianza y de Atención del factor Control de Estrés del CPRD que, en ambos casos, experimentan un aumento tras la inter-

Tabla 1. Programa de intervención psicológica aplicado

\begin{tabular}{|c|c|c|}
\hline SESIÓN & CONTENIDOS & ACTIVIDADES PERÍODO INTER-SESIONES \\
\hline 1 & - Evaluación: CPRD y entrevista & $\begin{array}{l}\text { - Valoración Subjetiva de mi Perfil Psicológico: Puntos fuertes y } \\
\text { débiles (PDF) }\end{array}$ \\
\hline 2 & $\begin{array}{l}\text { - Análisis CPRD y Análisis de PFD } \\
\text { - Explicación cumplimentación auto-registros } \\
\text { - Explicación Ejercicio Atención }\end{array}$ & $\begin{array}{l}\text { - Objetivos Temporada } \\
\text { - Errores competición } \\
\text { - Registro Calidad Entrenos } \\
\text { - Ejercicio de Atención }\end{array}$ \\
\hline 3 & $\begin{array}{l}\text { - Explicación Pautas Generales Competición } \\
\text { - Análisis errores competición } \\
\text { - Análisis Registro Calidad Entrenos } \\
\text { - Seguimiento Atención } \\
\text { - Explicación Objetivos para el Partido y Plan de Competición }\end{array}$ & $\begin{array}{l}\text { - Objetivos Partido } \\
\text { - Plan Competición } \\
\text { - Ejercicio de Atención } \\
\text { - Registro errores competición }\end{array}$ \\
\hline 4 & $\begin{array}{l}\text { - Análisis Objetivos Partido } \\
\text { - Análisis Plan Competición } \\
\text { - Seguimiento Atención } \\
\text { - Explicación Autoconfianza y técnica de autoinstrucciones }\end{array}$ & $\begin{array}{l}\text { - Objetivos Partido } \\
\text { - Plan Competición } \\
\text { - Autoinstrucciones competición }\end{array}$ \\
\hline 5 & $\begin{array}{l}\text { - Análisis Objetivos Partido } \\
\text { - Análisis Plan Competición } \\
\text { - Análisis autoinstrucciones } \\
\text { - Explicación Visualización y Evaluación capacidad visualizar }\end{array}$ & $\begin{array}{l}\text { - Objetivos Partido } \\
\text { - Plan Competición } \\
\text { - Entrenamiento visualización (registros) }\end{array}$ \\
\hline 6 & $\begin{array}{l}\text { - Análisis registros capacidad visualizar } \\
\text { - Entrenamiento visualización }\end{array}$ & $\begin{array}{l}\text { - Objetivos Partido } \\
\text { - Plan Competición } \\
\text { - Entrenamiento visualización (registros) }\end{array}$ \\
\hline 7 & $\begin{array}{l}\text { - Análisis registros capacidad visualizar } \\
\text { - Entrenamiento visualización (Fase } 2^{a} \text { y 3a) } \\
\text { - Registros Objetivos y Plan Competición }\end{array}$ & $\begin{array}{l}\text { - Objetivos Partido } \\
\text { - Plan Competición } \\
\text { - Entrenamiento visualización: registros Fase } 2^{a} \text { y } 3^{a}\end{array}$ \\
\hline 8 & $\begin{array}{l}\text { - Análisis registros capacidad visualizar } \\
\text { - Entrenamiento visualización (Fase } 2^{a} \text { y } 3^{a} \text { ) }\end{array}$ & $\begin{array}{l}\text { - Cumplimentación CPRD } \\
\text { - Valoración Subjetiva de mi Perfil Psicológico: Puntos fuertes y } \\
\text { débiles (PFD) }\end{array}$ \\
\hline 9 & $\begin{array}{l}\text { - Evaluación Final } \\
\text { - Valoración Programa } \\
\text { - Perspectivas de futuro }\end{array}$ & \\
\hline
\end{tabular}


vención. Respecto a la Autoconfianza el jugador parece haber mejorado tanto en su confianza general hacia la práctica del fútbol ("mi confianza en mí mismo es ahora más fuerte y estable, ningún contratiempo como cualquier pequeña lesión o un mal entrenamiento la va a debilitar", "tengo más fe en mí mismo"), como la referida a la competición ("en la mayoría de los partidos confío en que lo haré bien"; "no tengo apenas dudas respecto a mis posibilidades de hacerlo bien en un partido", "aunque comience mal un partido sigo confiando en mí mismo"). Respecto a la Atención el jugador también parece haber mejorado, sobre todo en el mantenimiento atencional y focalización durante los partidos ("durante mi actuación en un partido consigo centrar mi atención en lo que tengo que hacer", "me concentro con facilidad en aquello que es lo más importante en cada momento del partido", "cuando cometo un error consigo concentrarme rápidamente en lo que tengo que hacer").
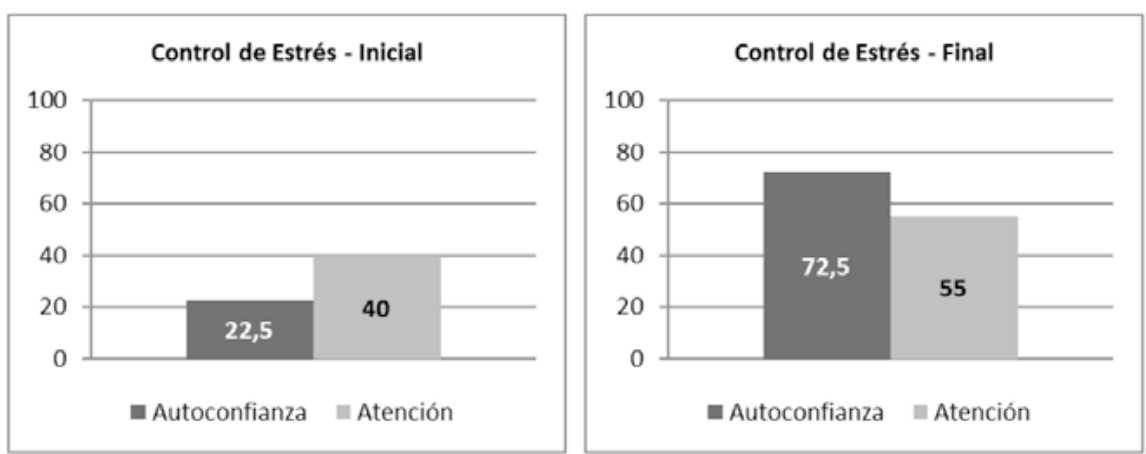

Figura 1. Valores de Autoconfianza y Atención del factor Control de Estrés.

En la Figura 2 se pueden observar los valores de Autoconfianza y de Atención del factor Influencia de la Evaluación del Rendimiento del CPRD, en el que se aprecia un aumento importante en las dos variables. Respecto a la Autoconfianza el jugador considera que su nivel de confianza para un partido no depende como antes de los éxitos o fracasos en partidos anteriores, gestionando mejor la presión e influencia de los resultados sobre el rendimiento. Respecto a la Atención el jugador también parece haber mejorado, sobre todo al poder mantener la concentración en el partido a pesar de no estar jugando bien.

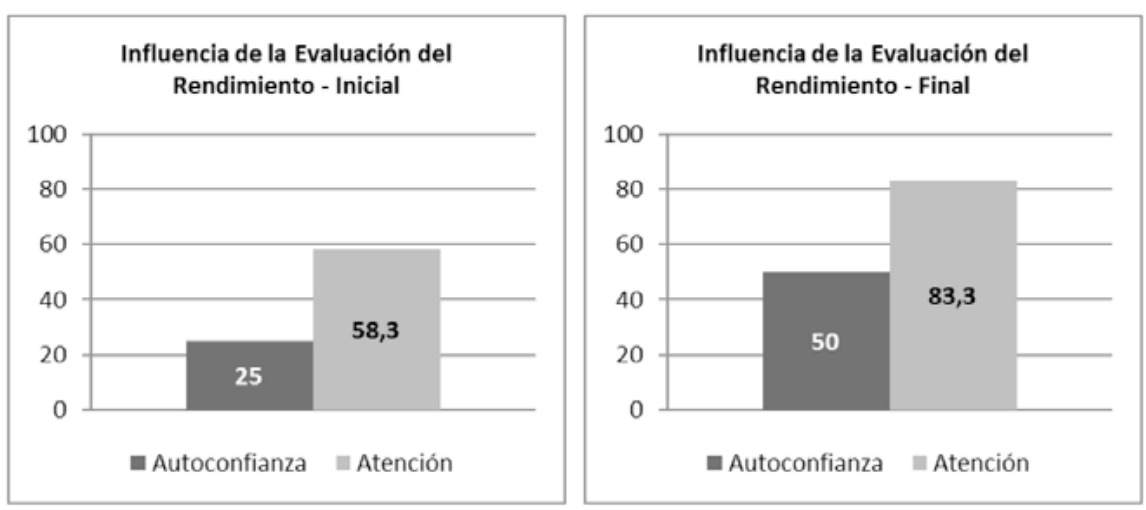

Figura 2. Valores de Autoconfianza y Atención del factor Influencia de la Evaluación del Rendimiento.
En la Figura 3 se pueden observar los valores que el CPRD muestra en relación a la Atención en tres aspectos relacionados con esta función: habilidad para estar concentrado, falta de habilidad general y motivos específicos para la pérdida de concentración. Se aprecia un aumento en los tres. Respecto a la habilidad para estar concentrado el jugador considera que se concentra con mayor facilidad en aquello que es lo más importante en cada momento de un partido y que durante los entrenamientos suele estar muy concentrado en lo que tiene que hacer. Respecto a la falta de habilidad general considera que no suele tener problemas para concentrarse mientras compite y que durante su participación en un partido puede focalizar su atención en lo verdaderamente importante. Respecto a los motivos específicos para la pérdida de concentración considera que no pierde ésta aunque perciba que lo hace mal, ni cuando comete errores.
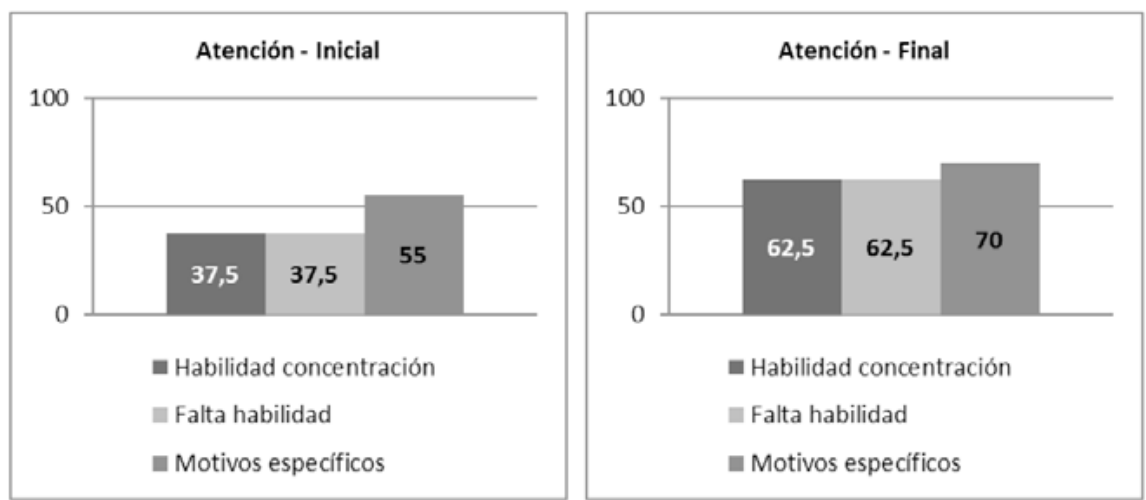

Figura 3. Valores de Atención en tres aspectos clave de ésta del CPRD.

En la Figura 4 se pueden observar los valores que el CPRD muestra respecto a la Autoconfianza en tres aspectos relacionados con esta variable: confianza general, competición y entrenamientos. Se aprecia un aumento en los tres. Respecto a la confianza general el jugador considera que tiene bastante más confianza y fe en sí mismo que anteriormente, y que aquélla es más estable. Respecto a la confianza competitiva (durante la competición) considera que en la mayoría de los partidos confía en que lo hará bien, con muy pocas dudas, y además confía en seguir haciendo un buen partido aunque comience mal, o se trate de una de sus peores actuaciones, confiando en sí mismo aún en los momentos más difíciles de un partido. Respecto a los entrenamientos considera que suele mantener muy bien la concentración en aquello que es lo más relevante.

En la Figura 5 se pueden observar los valores que el CPRD muestra respecto a la Tolerancia al bajo rendimiento del deportista en tres aspectos: malas actuaciones, errores y críticas. Se aprecia un aumento en dos de estos tres aspectos, en malas actuaciones y en errores, manteniendo el valor inicial en críticas (que en ambos casos, inicial y final es medio alto, con un valor del 75\%). Respecto a las malas actuacio- 


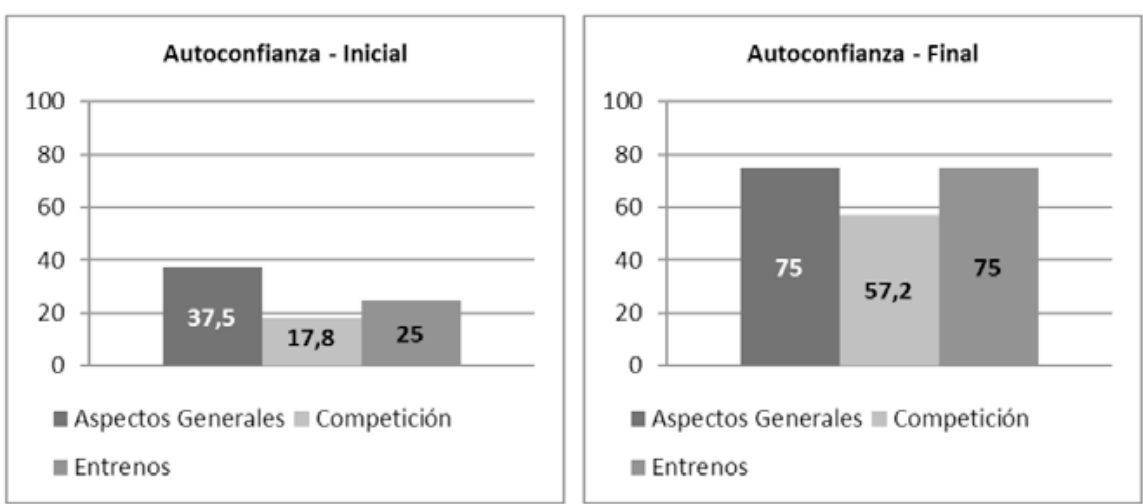

Figura 4. Valores de Autoconfianza en tres aspectos clave de ésta del CPRD.

nes el jugador considera que ha mejorado en confianza ya que ésta no se resiente aunque comienza mal un partido, o aunque lo esté jugando mal, además en estos casos no pierde la concentración ni le preocupa lo que piensen los otros (entrenador, compañeros, público, etc.). Respecto a los errores considera que ha mejorado mucho ya que ahora no se pone ansioso y además consigue olvidarse rápidamente del error y concentrarse en lo que tiene que hacer.

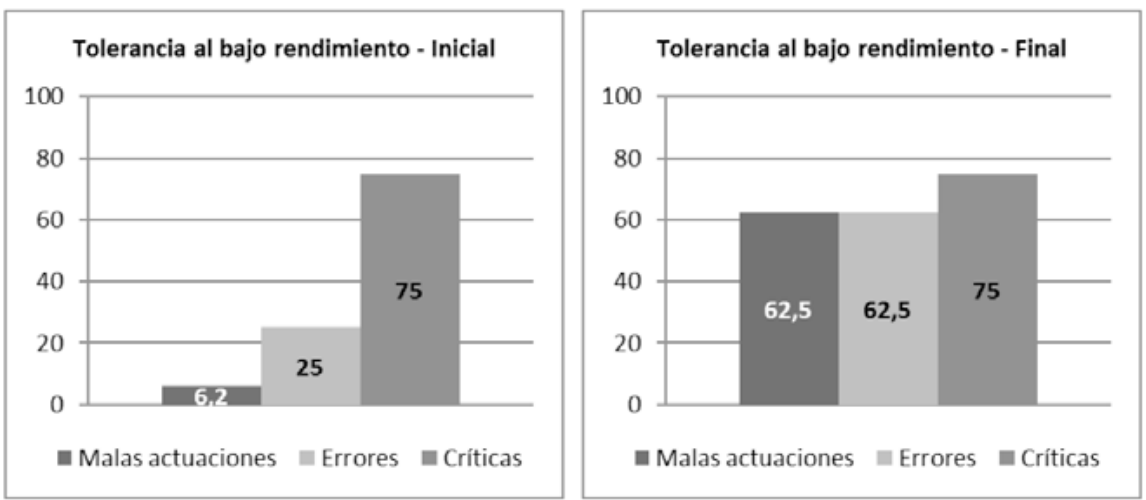

Figura 5. Valores de Toleracia al bajo rendimiento en tres aspectos clave de ésta del CPRD.

Respecto a la valoración realizada por el deportista a través del Cuestionario de Evaluación de la Preparación Psicológica se le sugirió hacer ésta tanto cuantitativa (en una escala de 0 a 10 , donde 0 = nada y 10 = mucho) como cualitativamente (análisis de contenido de respuesta). En la Tabla 2 se puede observar las respuestas del sujeto.

\section{Tabla 2. Cuestionario de Evaluación de la Preparación Psicológica}

\section{¿Estás satisfecho con la preparación psicológica realizada?: 9}

Indica los aspectos que más te han gustado y razona porqué

Los aspectos que más me han gustado ha sido la preparación de los partidos, ya que la mente se me llenaba de pensamientos negativos, y aunque no esté ya del todo preparado, sí que los encaro con más confianza. De manera que los objetivos sobre todo, hacen que minimice mucho el partido y me centre solo en lo que tengo que hacer. También quiero sacarle más partido a la visualización ya que se me da bastante bien y quiero poder preparar partidos y entrenamientos, también encarar nuevas experiencias de cara al año que viene, al nuevo vestuario y nuevas personas.

Indica los aspectos que menos te han gustado y razona porqué

Una técnica que no he utilizado mucho fue la de escribir cada entrenamiento y como lo he realizado, ya que para esta etapa donde voy más liado con la universidad, no quería tampoco sobrecargarme de "deberes" a realizar. No descarto en un futuro cuando solo me dedique a entrenar, poder llevar a cabo un seguimiento de cada entrenamiento. Las dificultades en un partido también me ha costado llevarlas, o por lo menos intentaré hacer menos, ya que me cuesta almacenar 3 dificultades en un partido y 3 alternativas,ii no las recuerdo!!

\section{¿En qué medida crees que te ha servido para tu preparación deportiva general?: 8}

Indica los aspectos que más te han gustado y razona porqué

Sí considero que me haya servido, de hecho el entrenador me comentaba que había visto un cambio de enero hacia estas fechas, de intensidad, de concentración y sobre todo de no centrar todo al fútbol. Ya te digo que no considero que sea un fiera mentalmente, al contrario, porque me sigo encabronando con algunas situaciones, pero sí es verdad que intento relativizar todo un poco e intentar disfrutar más del proceso. Estoy seguro que tengo las cualidades y que con la mentalidad más positiva puedo rendir más, es decir, la confianza. Aquí me gustaría trabajar más con objetivos, ya que suelo pensar mucho en el futuro y me olvido del presente. Me gusta tener motivación cada semana y creo que trabajaría bien con objetivos.

Indica los aspectos que menos te han gustado y razona porqué

Aquí coloco la técnica del seguimiento de cada entrenamiento, que ya lo he explicado anteriormente.

\section{¿En qué medida crees que te ha servido para la competición deportiva?: 10}

\section{Indica los aspectos que más te han gustado y razona porqué}

Creo que me ha salvado bastante el haber trabajado con objetivos en partidos decisivos, ya que no tenía ninguna técnica ni recurso para afrontar los partidos. De manera que estoy muy satisfecho con lo trabajado porque de no ser así no sé qué hubiese ocurrido. Los objetivos para cada partido me han resultado muy interesantes porque he podido minimizar al máximo cada partido, evadiéndome de todo lo que rodea. Aun así, puedo mejorar muchísimo la técnica ya que a veces inconscientemente desconectaba.

Indica los aspectos que menos te han gustado y razona porqué

Respecto a los partidos, creo que como he mencionado anteriormente, las dificultades debido a tener que recordar 6 aspectos, 3 dificultades y 3 planes alternativos. Tendré que reducirlo. 
Tabla 2 (Continuación)

\section{Valora la satisfacción y la eficacia de las técnicas y estrategias utilizadas}

\begin{tabular}{|c|c|c|c|c|c|c|c|c|c|c|}
\hline \multirow{2}{*}{ Técnicas/Estrategias } & \multicolumn{10}{|c|}{ Valoración cuantitativa SATISFACCIÓN } \\
\hline & 0 & 1 & 2 & 3 & 5 & 6 & 7 & 8 & 9 & 10 \\
\hline Reg. errores compet. & & & & & & & & $x$ & & \\
\hline Reg. Calidad Entren. & & & $x$ & & & & & & & \\
\hline Ejercicio Atención & & & & & $x$ & & & & & \\
\hline Pautas Generales Comp & & & & & & & & & $x$ & \\
\hline Obj. para el Partido & & & & & & & & & & $x$ \\
\hline Plan de Competición & & & & & & $x$ & & & & \\
\hline Visualización & & & & & & & & & & $x$ \\
\hline
\end{tabular}

\begin{tabular}{|c|c|c|c|c|c|c|c|c|c|c|}
\hline \multirow{2}{*}{ Técnicas/Estrategias } & \multicolumn{8}{|c|}{ Valoración cuantitativa EFICACIA } & \multirow[b]{2}{*}{9} & \multirow[b]{2}{*}{10} \\
\hline & 0 & 1 & 2 & 3 & 5 & 6 & 7 & 8 & & \\
\hline Reg. errores compet & & & & & & & & & & $x$ \\
\hline Reg. Calidad Entren. & & $x$ & & & & & & & & \\
\hline Ejercicio Atención & & & & & $x$ & & & & & \\
\hline Pautas Generales Comp & & & & & & & & & $x$ & \\
\hline Obj. para el Partido & & & & & & & & & $x$ & \\
\hline Plan de Competición & & & & & & & & & & $x$ \\
\hline Visualización & & & & & & & & & & $x$ \\
\hline
\end{tabular}

\section{Valoración cualitativa EFICACIA}

(indica lo que consideres más representativo de cada técnica o estrategia; si la has utilizado mucho o no, si te ha resultado sencilla o no, y cualquier otro aspecto que consideres importante)

Registro errores competición Me ha parecido súper eficaz, ya que alguna vez vimos que se me cruzaban los cables entrenando y posteriormente me ha pasado lo mismo y he sabido controlarme.

Registro Calidad Entrenos Esta es la que menos debido al tiempo en hacerla, creo que se puede hacer algo repetitivo todos los días.

Ejercicio Atención Me ha parecido bien pero no la he utilizado mucho, creo que debería trabajarla más.

Pautas Generales Competición Al principio me aportó muchísimo ya que no tenía ninguna herramienta de este tipo, me gustaría tener algunas más, de futbolistas que tengan su propio sistema de concentración, más información.

Objetivos para el Partido Es la que más, junto a la visualización por todo lo que he comentado anteriormente.

Plan de Competición Me parece bien, el día de antes decir, a ver mañana ¿Qué voy a hacer? Me parece genial situarme en el partido y ver como lo planteo

Visualización Se me da muy bien y quiero sacarle más partido del que le doy.

Nota: Las abreviaturas de la Valoración cuantitativa (Satisfacción y Eficacia) se refieren a las mismas Técnicas/Estrategias que se pueden observar completas en la Valoración cualitativa

\section{Discusión}

Los resultados confirman la hipótesis planteada ("el futbolista mejorará su capacidad de concentración y su nivel de autoconfianza tras la aplicación de un programa de entrenamiento psicológico específico"). La observación de la evaluación psicológica final comparada con la inicial del futbolista parece indicar que, efectivamente, éste ha conseguido mejorar sus recursos psicológicos aplicados al ámbito deportivo, tanto en la competición como en los entrenamientos. De hecho, una de las claves para el éxito deportivo es, precisamente, el disponer de un buen repertorio de habilidades y de recursos psicológicos que le permitan al deportista gestionar adecuadamente los requerimientos 
del contexto. En este caso, el trabajo se centró en la mejora de la autoconfianza y la concentración del jugador; ambas son dos variables citadas por los propios deportistas como fundamentales en su rendimiento (Weinberg y Gould, 2010), y por lo tanto objeto de intervención psicológica (Latinjak, Torregrosa y Renom, 2009; Mora, Zarco y Blanca, 2001).

Desde la perspectiva de una gestión adecuada del estrés propio de la competición y del entrenamiento, el jugador ha mostrado una mejoría importante, ya que se ve con un nivel de confianza bastante más alto, tanto a nivel general (confianza en sí mismo) como en el desarrollo de los partidos (confianza en sus posibilidades y asunción positiva de errores); y parece evidenciar una mejor capacidad de concentración (concentración en lo relevante, no anclaje en los errores). Por otro lado, desde la perspectiva de la presión que genera la influencia de la evaluación de su rendimiento, el jugador también ha mostrado una significativa mejoría, ya que se ve con una confianza más estable y consolidada, no dependiente de resultados anteriores, y puede mantener la concentración durante el partido a pesar de no estar jugando bien.

En general se puede indicar que el jugador ha mejorado su capacidad de concentración y su nivel de autoconfianza. Respecto a la primera por haber adquirido mayor habilidad para concentrarse y poder hacerlo tanto en los partidos como en los entrenamientos, sabiendo diferenciar los estímulos relevantes de los que no lo son, además de reinterpretar los errores desde una perspectiva diferente, percibiéndolos como algo natural de la práctica deportiva. Respecto a la autoconfianza por considerarse con mayor confianza general, y competir con mayor seguridad en sí mismo y en sus posibilidades. Además, los datos obtenidos en Tolerancia al bajo rendimiento vienen a confirmar lo anterior, ya que el jugador considera que las malas actuaciones, cuando juega mal un partido o lo está jugando no merma como antes su confianza, ni pierde la concentración, ni le preocupa lo que piensen los demás, lo que hace mejorar su rendimiento y satisfacción personal.

Respecto a la evaluación que el sujeto hace del programa de intervención psicológica, en general se puede afirmar que su satisfacción y su utilidad respecto al mismo son muy buenas. En línea con Page y Thelwell (2013) se puede considerar que la "validación social", es decir la evaluación que realizan los sujetos sobre la formación recibida, es básica, usándose además de para determinar la satisfacción con la intervención y la eficacia de la misma (Olmedilla, Ortega, Ortín y Andreu, 2010), para proporcionar datos válidos para futuras intervenciones. Diferentes autores (Neil, Hanton y Mellalieu, 2013; Olmedilla et al., 2008) consideran que algunas de las formas que pueden servir para evaluar este pro- ceso de formación psicológica son el índice de satisfacción que tienen los deportistas respecto al programa recibido y la evaluación específica que realizan sobre el mismo, procedimiento necesario para validar la intervención psicológica.

En este caso, se puede considerar que el jugador está muy satisfecho con el programa recibido (señalando como claves algunas de las técnicas y estrategias utilizadas, como el establecimiento de objetivos para el partido, o la visualización), que le ha servido en gran medida para su preparación deportiva general (disposición cognitiva diferente del contexto deportivo, mayor relativización de algunas "verdades futbolísticas") y que le ha resultado fundamental para su participación en los partidos (el trabajo con objetivos le ha ayudado a concentrarse mejor, y por ende a tener más confianza en lo que hace; el registro de errores le ha servido para abordar éstos de manera diferente, tanto racional como emocionalmente). Ratificando alguno de los aspectos vivenciales de los experimentos de comportamiento (Bennett-Levy, 2003), en los que la planificación de tareas proporciona al sujeto una experiencia que pone a prueba su creencia, impulsando la construcción de nuevas creencias más adaptativas y contribuir aún más al desarrollo de la formulación (Bennett-Levy et al., 2004; Turner, Slater y Barker, 2014).

La valoración de estrategias y técnicas, tanto a nivel de satisfacción como de eficacia, permite señalar que el jugador con algunas estrategias (registro de errores durante el partido, plan de competición) no está totalmente satisfecho (8 para la primera; 6 para la segunda), y sin embargo sí las considera muy eficaces (10 en ambos casos). También es reseñable el valor que le otorga a la práctica de la visualización (10 en satisfacción y en eficacia), lo que de alguna manera ratifica la importancia de esta técnica en los programas de entrenamiento psicológico, tal y como indican diferentes autores (De la Vega, 2002; Jordet, 2005; Nordin y Cumming, 2008; Rodríguez y Galán, 2007; Stewart y Hall, 2016; Wright, McCormick, Birks, Loporto y Holmes, 2015).

Por último, indicar que la presentación de estudios de caso es muy necesaria, pero tal y como señalan McArdle y Moore (2012), y desde la perspectiva cognitivo-conductual, sería importante desarrollar un sistema basado en la evidencia para orientar a los profesionales de la Psicología del Deporte que adoptan esta perspectiva. Algunos autores (Bieling y Kuyken, 2003) recomiendan que los estudios de caso cumplan los criterios de fiabilidad (los psicólogos del deporte ¿pueden presentar de forma fiable sus casos?, calidad (los estudios de caso ¿existe coherencia entre los constructos clave utilizados y las áreas problemáticas del deportista?) y resultado (el estudio de casos ¿conduce a la mejora de resultados de la intervención?). 


\section{Referencias}

Abenza, L., Bravo, J.F. y Olmedilla, A. (2006). Estrategias psicológicas para una intervención en crisis: un caso de balonmano femenino. Revista de Psicología del Deporte, 15, 109-115.

Abenza, L., González, J., Reyes, L., Reyes, F., Blas, A. y Olmedilla, A. (2014). Descripción y evaluación del entrenamiento psicológico de una deportista de regata clase laser radial. Revista Iberoamericana de Psicología del Ejercicio y el Deporte, 9, 67-92.

Barker, J.B., Mellalieu, S.D., McCarthy, P.J., Jones, M.V. y Moran, A. (2013). A Review of Single-Case Research in Sport Psychology 1997-2012: Research Trends and Future Directions. Journal of Applied Sport Psychology, 25, 4-32.

Bennett-Levy, J. (2003). Mechanisms of change in cognitive therapy: The case of automatic thought records and behavioral experiments. Behavioural and Cognitive Psychotherapy, 31, 261-277.

Bennett-Levy, J., Westbrook, D., Fennell, M., Cooper, M., Rouf, K. y Hackmann, A. (2004). Behavioural experiements: historical and conceptual underpinnings. En J. Bennett-Levy, G. Butler, M. Fennell, A. Hackman, M. Mueller y D. Westbrook (Eds.), Oxford guide to behavioural experiments in cognitive therapy, (pp. 21-58). Oxford, UK: Oxford University Press.

Bieling, P.J. y Kuyken, W. (2003). Is cognitive case formulation science or science fiction? Clinical Psychology: Science and Practice, 10, 52-69.

Buceta, J.M. (1998). Psicología del entrenamiento deportivo. Madrid, España: Dykinson.

Buceta, J.M., López de la Llave, A., Pérez-Llantada, M.C., Vallejo, M. y del Pino, M.D. (2002). Intervención psicológica con corredores de maratón: características y valoración del programa aplicado en el maratón de Madrid. Revista de Psicología del Deporte, 17, 83-109.

Cantón, E., Checa, I. y Ortín, F.J. (2009). Intervención psicológica con un nadador de medio acuático abierto. Cuadernos de Psicología del Deporte, 9(2), 57-65.

Chicau, C., Silva, C. y Palmi, J. (2012). Programa de intervención psicológica para la optimización del concepto de equipo (team building) en jóvenes futbolistas. Revista de Psicología del Deporte, 21, 49-58.

Cox, R.H. (2008). Psicología del deporte: conceptos y sus aplicaciones. Madrid, España: Ed. Médica Panamericana.

De la Vega, R. (2002). Desarrollo de un programa de entrenamiento en visualización/imaginación con futbolistas. Revista digital efdeportes, 8, 46. Recuperado de http://www.efdeportes.com/ efd46/visual7.htm

García-Naveira, A. y Jerez, P. (2012). Departamento de psicología del club Atlético de Madrid: filosofía, programación y desempeño profesional en el fútbol base. Cuadernos de Psicología del Deporte, 12(1), 111-120.

Gimeno, F. y Buceta, J.M. (2010). Evaluación psicológica en el deporte. El cuestionario CPRD. Madrid, España: Dykinson.

Gimeno, F., Buceta, J.M. y Pérez-Llantada, M. (2001). El cuestionario "Características Psicológicas Relacionadas con el Rendimiento Deportivo" (CPRD): Características psicométricas. Análise Psicológica, 7(XIX), 93-133.

Gimeno, F., Buceta, J.M. y Pérez-Llantada, M.C. (2007). Influencia de las variables psicológicas en el deporte de competición: evaluación mediante el cuestionario Características Psicológicas relacionadas con el Rendimiento Deportivo. Psicothema, 19, 666-671.

Godoy-Izquierdo, D., Vélez, M. y Godoy, J. (2007). Results of a seasonal-period psychological skills training in football young-players. 12th European Congress of Sport Psychology. Halkidiki, Greece.

Jaenes, J.C., Caracuel, J.C. y Peñaloza, R. (2012). Intervención en psicología del deporte: un caso de remo de alta competición. Revista de Psicología del Deporte, 21, 59-64.

Jordet, G. (2005). Perceptual training in soccer: An imagery intervention study with elite players. Journal of Applied Sport Psychology, 17, 140-156.

Junichi, M. y Hajime, K. (2007). Psychological skill training for the Japanese soccer team in 2005 Universiade game in Izmir. Journal of Sport Science and Medicine, 6(suppl. 10), 88.

Kuntz, S. y Hessler, A. (1998). Bridging the gap between theory and practice: Foster- ing active learning through the case study method. Presentation at the Association of American Colleges and Universities (AACU) Annual Meeting. Washington, D.C.

Lameiras, J., Almeida, P., Pons, J. y García-Mas, A. (2014). Incorporación de una rutina para la optimización del rendimiento deportivo. Revista de psicología del deporte, 23, 337-343.

Landi, S., Benedetti, S. y Merla, G. (2007). The goalkeepers' bravery: A self-efficacy study. 12th European Congress of Sport Psychology. Halkidiki, Greece.

Latinjak, A. T., Font-Lladó, R., Zourbanos, N. y Hatzigeorgiadis, A. (2016). Goal-Directed Self-Talk Interventions: A Single-Case Study With an Elite Athlete. The Sport Psychologist, 30, 189-194.

Latinjak, A.T., Torregrosa, M. y Renom, J. (2009). Aplicando el auto-habla al tenis: su impacto sobre el foco atencional y el rendimiento. Cuadernos de Psicología del Deporte, 9(2), 19-29.

Lindern, D. (2016). Desenvolvimento de uma intervenção com foco preventivo baseada na terapia cognitivo-comportamental e na psicologia positiva para atletas de futebol adolescentes. (Tesis Dissertação de Mestrado). Portugal: Pontícia Universidade Católica do Rio Grande do Sul.

Lorenzo, J., Gómez, M. A., Pujals, C. y Lorenzo, A. (2012). Análisis de los efectos de un programa de intervención psicológica en jóvenes jugadores de baloncesto. Revista de Psicología del Deporte, 21, 43-48.

McArdle, S. y Moore, P. (2012). Applying evidence-based principles from CBT to sport psychology. The Sport Psychologist, 26, 299-310.

Mora, J.A., Zarco, J.A. y Blanca, M.J. (2001). Atención-concentración como entrenamiento para la mejora del rendimiento deportivo en jugadores profesionales de fútbol. Revista de Psicología del Deporte, 10, 49-65.

Morilla, M., Pérez, E., Gamito, J.M., Gómez, M.A., Sánchez, J.E. y Valiente, M. (2001). Desarrollo de un programa de preparación psicológica con los equipos de un club de fútbol de la primera división española. Revista digital efdeportes, 7, 42. Recuperado de http://www. efdeportes.com/efd42/preps.htm

Mostaan, M., Sanatkaran, A. y Bahari, S.M. (2015). The Effects of Behavioral Intervention on Mood in Disabled Professional Basketball Players. Journal of Novel Applied Sciences 4, 797-803.

Munroe-Chandler, K.J., Murphy, L., Hall, C.R. y Fishburne, G. (2007). Speed/accuracy trade-off: An explanation for soccer imagery intervention?. 12th European Congress of Sport Psychology. HaIkidiki, Greece. 
Neil, R., Hanton, S. y Mellalieu, S.D. (2013). Seeing Things in a Different Light: Assessing the Effects of a Cognitive-Behavioral Intervention upon the Further Appraisals and Performance of Golfers. Journal of Applied Sport Psychology, 25, 106-130.

Nieto, G. y Olmedilla, A. (2001). Planificación del entrenamiento psicológico en atletas de élite: un caso en marcha atlética. Revista de Psicología del Deporte, 10, 127-142.

Nordin, S.M. y Cumming, J. (2008). Exploring common ground: Comparing the imagery of dancers and aesthetic sport performers. Journal of Applied Sport Psychology, 20, 375-391.

Olivares, E.M., López, J.J. y Garcés de los Fayos, E.J. (2016). Entrenamiento psicológico en tiro olímpico: estudio de caso. Revista de Psicología del Deporte, 25, 131-135.

Olmedilla, A., Bazaco, M.J., Ortega, E. y Boladeras, A. (2011). Formación psicológica, satisfacción y bienestar percibido en futbolistas jóvenes. Revista Científica Electrónica de Psicología, 12, 221-237.

Olmedilla, A., Ortega, E., Boladeras, A., Ortín, F.J. y Bazaco, M.J. (2013). Entrenamiento en estrategias y técnicas psicológicas y percepción de ayuda en futbolistas juveniles. Revista Euroamericana de Ciencias del Deporte, 2(1), 51-58.

Olmedilla, A., Ortega, E., González, J. y Serpa, S. (2015). Psychological Training in Sailing: Performance Improvement for the Olympic Classification Phase. Universal Journal of Psychology, 3(4), 122-131.

Olmedilla, A., Ortega, E., Ortín, F. y Andreu, M.D. (2008). Entrenamiento psicológico en fútbol: satisfacción y aplicabilidad. Revista Iberoamericana de Psicología del Ejercicio y el Deporte, 3, 91-46.

Olmedilla, A., Ortega, E., Ortín, F.J. y Andreu, M.D. (2010). Programa de intervención psicológica en futbolistas: evaluación de habilidades psicológicas mediante el CPRD. Revista de Psicología del Deporte, 19, 249-262.

Page, J. y Thelwell, R. (2013). The Value of Social Validation in Single-Case Methods in Sport and Exercise Psychology. Journal of Applied Sport Psychology, 25, 61-71.

Reyes, M., Raimundi, M.J. y Gómez, L. (2012). Programa de entrenamiento en habilidades psicológicas en jugadoras de voleibol de alto rendimiento. Cuadernos de Psicología del Deporte, 12(1), 9-16.
Rodríguez, M.C. y Galán, S.T. (2007). Programa de entrenamiento en imaginería como función cognoscitiva y motivadora para mejorar el rendimiento deportivo en jóvenes patinadores de carreras. Cuadernos de Psicología del Deporte, 7(1), 5-24.

Romero, A.E., Zapata, R., García-Mas, A., Brustad, R.J., Garrido, R. y Letelier, A. (2010). Estrategias de afrontamiento y bienestar psicológico en jóvenes tenistas de competición. Revista de Psicología del Deporte, 19, 117-133.

Salgado, R., Rivas, C.A. y García-Mas, A. (2011). Aparición del burnout en jugadores de fútbol de primera división profesional en México: estrategias de intervención. Cuadernos de Psicología del Deporte, 17(2, Supl.), 57-62.

Sheard, M. y Golby, J. (2006). Effect of a psychological skills training program on swimming performance and positive psychological development. International Journal of Sport and Exercise Psychology, 4, 149-169.

Stewart, N.W. y Hall, C. (2016). The Effects of Cognitive General Imagery Training on Decision Making Abilities in Curling: A Single-Subject Multiple Baseline Approach. Journal of Applied Sport Psychology, doi: http://dx.doi.org/10.1080/10413200.201 6.1213331

Turner, M. y Barker, J.B. (2013). Examining the Efficacy of Rational-Emotive Behavior Therapy (REBT) on Irrational Beliefs and Anxiety in Elite Youth Cricketers. Journal of Applied Sport Psychology, 25, 131-147.

Turner, M.J., Slater, M.J. y Barker, J.B. (2014). Not the end of the world: The effects of rational-emotive behavior therapy (REBT) on irrational beliefs in elite soccer academy athletes. Journal of Applied Sport Psychology, 26, 144-156.

Weinberg, R.S. y Gould, D. (2010). Fundamentos de Psicología del Deporte y del Ejercicio físico. Madrid: Ed. Médica Panamericana.

Wood, G. y Wilson, M.R. (2012). Quiet-eye training, perceived control and performing under pressure. Psychology of Sport and Exercise, 13, 721-728.

Wright, D.J., McCormick, S.A., Birks, S., Loporto, M. y Holmes, P.S. (2015). Action observation and imagery training improve the ease with which athletes can generate imagery. Journal of Applied Sport Psychology, 27, 156-170. 
Anexo I. Valoración subjetiva de mi perfil psicológico: puntos fuertes y débiles (ejemplo)

\begin{tabular}{|c|c|c|c|}
\hline \multicolumn{2}{|c|}{ ASPECTOS PSICOLÓGICOS FUERTES } & \multicolumn{2}{|c|}{ ASPECTOS PSICOLÓGICOS DÉBILES } \\
\hline Factor Psicológico & Ejemplo deportivo & Factor Psicológico & Ejemplo deportivo \\
\hline \multirow[t]{3}{*}{ Mentalidad ganadora } & $\begin{array}{l}\text { Me considero un deportista } \\
\text { con mentalidad ganadora, por- } \\
\text { que nunca me doy por vencido } \\
\text { y pase lo que pase lucho hasta } \\
\text { el final y pienso que siempre } \\
\text { donde he jugado he tenido } \\
\text { buenos resultados. }\end{array}$ & $\begin{array}{l}\text { No me siento un futbolista } \\
\text { importante en un partido. No } \\
\text { siento que pueda cambiar el } \\
\text { trayecto del equipo, no hago } \\
\text { por que ocurran cosas (en } \\
\text { ciertas ocasiones). Trato de } \\
\text { no fallar más que arriesgarme } \\
\text { a hacer por que las cosas } \\
\text { ocurran. }\end{array}$ & $\begin{array}{l}\text { Cuando juego un partido, en } \\
\text { muchas ocasiones, trato de } \\
\text { hacerlo de tal forma de que } \\
\text { no falle, y cuando destaca un } \\
\text { futbolista es cuando tiene la } \\
\text { confianza de fallar y volver } \\
\text { a fallar. Tengo calidad para } \\
\text { sacar las faltas y córner pero } \\
\text { cuando he sacado alguno en } \\
\text { el partido, cuando hay tanta } \\
\text { gente mirándome la lanzo } \\
\text { para no fallar y no quedar } \\
\text { mal, es decir, no la lanzo con } \\
\text { confianza y entonces el balón } \\
\text { sale templado. Cuando entre- } \\
\text { no y ensayo centros y faltas, } \\
\text { como no tengo presión le pego } \\
\text { realmente bien, porque es así } \\
\text { como sé pegarle. }\end{array}$ \\
\hline & En otro aspecto de mi vida & & En otro aspecto de mi vida \\
\hline & $\begin{array}{l}\text { En la universidad, donde la } \\
\text { compatibilidad de horarios es } \\
\text { bastante difícil, siempre hablo } \\
\text { con los profesores y llego a un } \\
\text { acuerdo. Pese a la dificultad } \\
\text { de las circunstancias, intento } \\
\text { sacar todo adelante. Diría que } \\
\text { siempre pienso que me van a } \\
\text { ir bien las cosas. }\end{array}$ & & $\begin{array}{l}\text { En otros aspectos de mi vida } \\
\text { no lo siento así, suelo se más } \\
\text { tranquilo y no me afecta fallar. } \\
\text { En la universidad suelo hablar } \\
\text { en público y no tengo ningún } \\
\text { problema, creo que se refleja } \\
\text { más en el fútbol. }\end{array}$ \\
\hline
\end{tabular}

